\title{
The Intelligent Room for Elderly Care
}

\author{
Oscar Martinez Mozos ${ }^{1}$, Tokuo Tsuji ${ }^{2}$, Hyunuk Chae ${ }^{2}$, Shunya Kuwahata ${ }^{2}$, \\ YoonSeok $\mathrm{Pyo}^{2}$, Tsutomu Hasegawa ${ }^{2}$ Ken'ichi Morooka ${ }^{2}$, and Ryo Kurazume ${ }^{3}$ \\ 1 Laboratory for Image and Media Understanding, Kyushu University, \\ Fukuoka 819-0395, Japan \\ omozos@irvs.ait.kyushu-u.ac.jp, \\ WWW home page: http://www.informatik.uni-freiburg.de/ omartine/ \\ 2 Laboratory for Intelligent Robots and Vision Systems, Kyushu University, \\ Fukuoka 819-0395, Japan
}

\begin{abstract}
Daily life assistance for elderly is one of the most promising and interesting scenarios for advanced technologies in the present and near future. Improving the quality of life of elderly is also some of the first priorities in modern countries and societies where the percentage of elder people is rapidly increasing due mainly to great improvements in medicine during the last decades. In this paper, we present an overview of our informationally structured room that supports daily life activities of elderly. Our environment contains different distributed sensors including a floor sensing system and several intelligent cabinets. Sensor information is sent to a centralized management system which processes the data and makes it available to a service robot which assists the people in the room. One important restriction in our intelligent environment is to maintain a small number of sensors to avoid interfering with the daily activities of people and to reduce as much as possible the invasion of their privacy. In addition we discuss some experiments using our real environment and robot.
\end{abstract}

Keywords: Quality of Life Technologies, Assisyive Robotics, Intelligent Room, Ambient Intelligence

\section{Introduction}

Inside the many applications related to quality of life technologies, elderly care is one of the most promising ones both in social and economic terms. Improving the quality of life of elderly is also some of the first priorities in modern countries and societies where the percentage of elder people is rapidly increasing due mainly to great improvements in medicine during the last decades.

Different intelligent systems are being developed to assist elderly in their daily life environment. The main idea of these scenarios is to gather information about the environment of the people so that an intelligent system can keep track of their actions and their surroundings and can act when the person needs some assistance. The help received by the person can be on demand or alternatively the system can decide by itself when to take an action in order to assist people [16, 


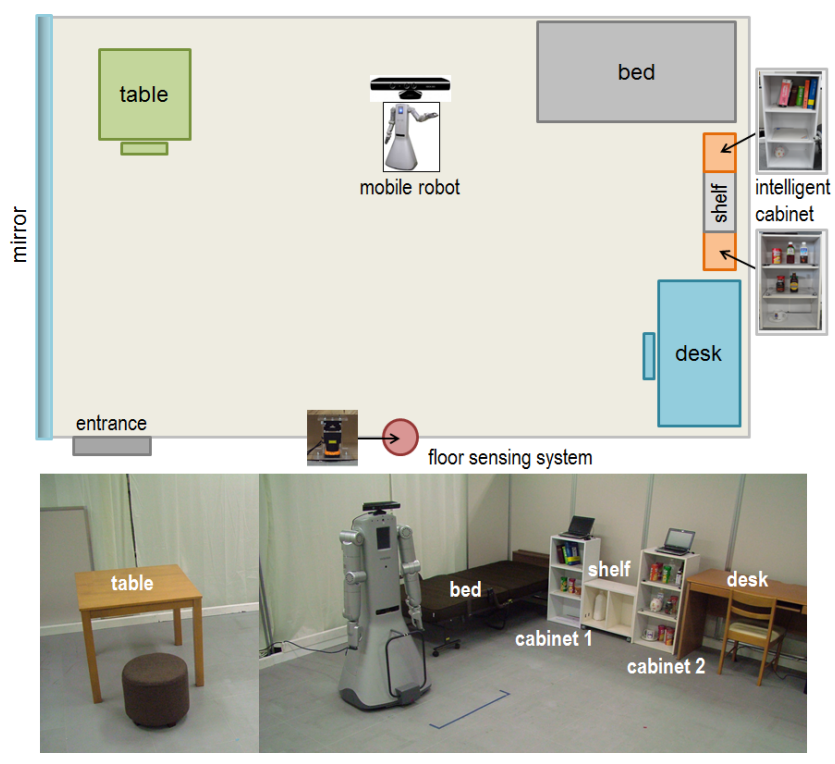

Fig. 1. The top image outlines a map of our intelligent room. Real images of the different components are show in the bottom images.

$7,4,8,13,14,11]$. In addition, service robots can be available to assist people alongside the intelligent environment. Actually, it is expected that service robots will soon be playing a role of companion to elderly people, or a role of assistant to humans with special needs at home [3], [17], [6], [2], [9], [5]. Moreover, one of the most demanding tasks by elderly users will be the go-and-fetch of objects that are needed for their everyday activities [18], [1].

This papers presents an overview of our informationally structured room which aims to assist elder people in their daily life. Our environment contains different distributed sensors including a floor sensing system and several intelligent cabinets as shown in Fig.1 Sensor information is sent to a centralized management system which processes the data and makes it available to a service robot which assists the people in the room. One important restriction in our intelligent environment is to maintain a small number of sensors to avoid interfering with the daily activity of people and to reduce as much as possible the invasion of their privacy. In addition we constrain the use of the camera on the robot to only some predetermined situations.

\section{The Informationally Structured Room}

This section briefly describes the different components of our informationally structured environment. In particular, our scenario represents a room in a house as shown in Fig. 1. The room contains two intelligent cabinets, one shelf, a bed, 

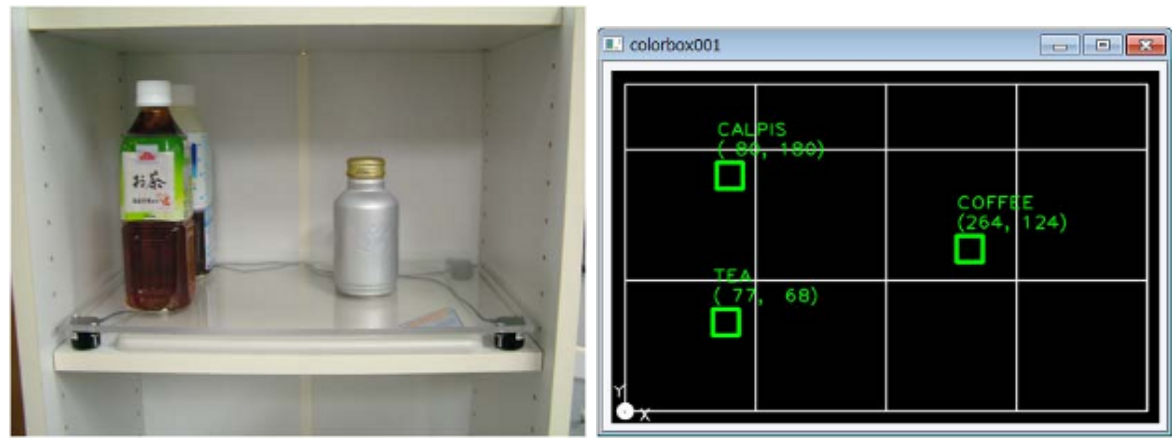

Fig. 2. Information about objects provided by the intelligent cabinet. Squares in the screen indicate the position of the different objects together with their description.

a desk with a chair, and a dining table. In addition, the room is equipped with a floor sensing system for object and people detection.

\subsection{Intelligent Cabinets}

The cabinets installed in our room (see Fig.1) are equipped with RFID readers and load cells to detect the type and position of objects inside. Every object in our environment is attached one RFID tag containing a unique ID that identifies the object. This ID is used to retrieve the attributes of the object in our database. Using the RFID readers we can detect the presence of a new object inside the cabinet. In addition, the information of the load cells allows us to determine its exact position inside the cabinet. An example detection of objects in one intelligent cabinet is shown in Fig. 2. Further details about our intelligent cabinets can be found in [10].

\subsection{Floor Sensing System}

In addition to the intelligent cabinets our room is equipped with a floor sensing system used to detect objects on the floor and people walking around. This sensing systems is composed of a laser range finder which is located on one side of the room as shown in Fig.1. Moreover, a mirror is installed along one side of the room to help the detection when clutter occurs. This configuration allows a reduction of dead angles of the LRF and it is more robust against occlusions [15]. An example detection of an object using this system is shown in Fig. 3.

People tracking is performed by first applying static background subtraction and then extracting blobs in the rest of the measurements. Blobs are later tracked by applying a Kalman filter by matching profiles of blobs corresponding to legs, and extending the motion using accelerations of legs [10]. 

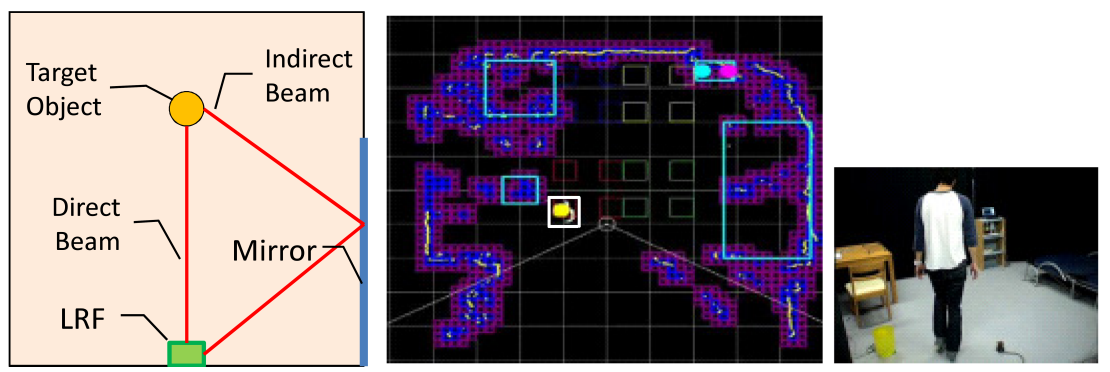

Fig. 3. The left image depicts an example of an object detected on the floor by our floor sensing system. The right image shows the detection of a person indicated by a white square.

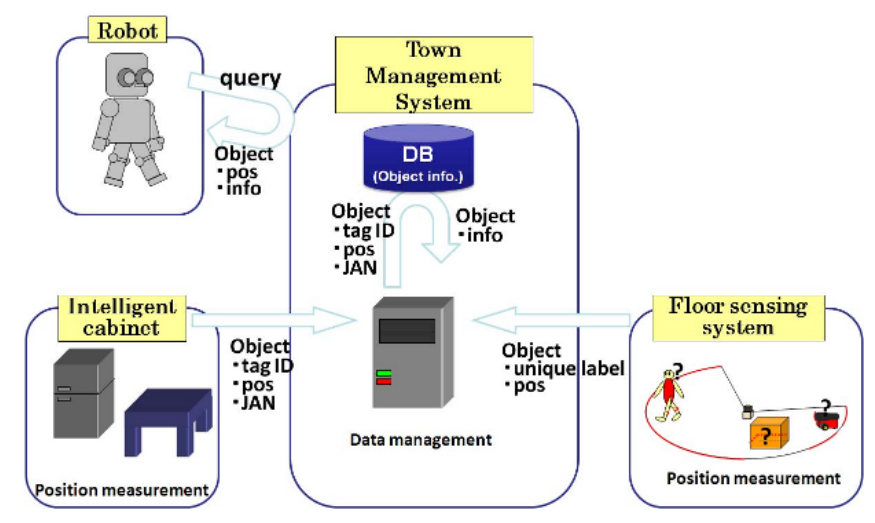

Fig. 4. Information flow between the TMS and the sensors and robot in our informationally strucutred room.

\subsection{Town Management System}

The previous sensing system and the robot itself are connected to our Town Management System (TMS), which integrates sensor data into an online environment database and provides robots with real-time information on its dynamically changing surroundings. The TMS was originally designed to include informationa about distributed sensors and robots in a wider environment [12]. This central database management system provides information about indoor maps, RFID tag ids and related information, and notification of predefined environment events and their occurrence. The information flow between our intelligent room and the TMS is shown in Fig. 4. 

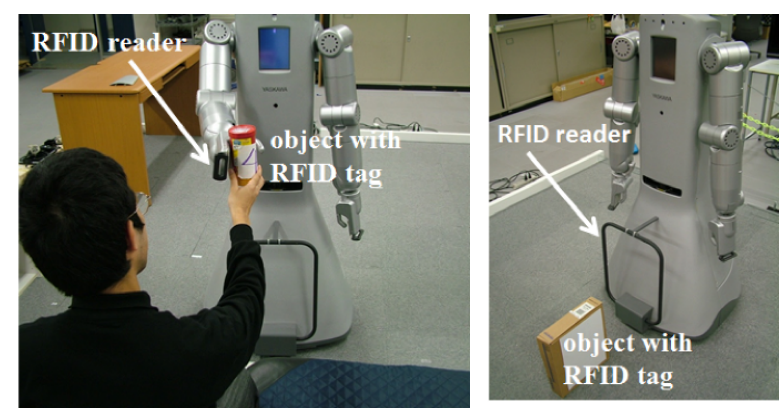

Fig. 5. Assistive humanoid robot SmartPal equipped with RFID readers.

\section{Service Robot}

Finally, the person acting in the room is assisted by a SmartPal humanoid robot (Fig. 5) from Yaskawa Electric Corporation. This robot will be responsible for fetching objects or pointing to them. The robot is composed of a mobile platform, two arms with seven joints, and one-joint grippers used as hands. In addition we equipped the robot with a RGB-D camera which is use for object recognition in restricted regions of interest, and only under specific requests. In order to maintain the privacy of people we do not use this camera for general vision purposes. Additional RFID readers are situated on the hands and front of the robot as shown in Fig. 5.

\subsection{Visual Memory for Object Searching}

Our service robot is equipped with a visual memory system which helps in the task of object searching and finding. The visual memory system is used by the robot to detect changes on predefined places where objects usually appear. In our case we restrict the application of this visual system to the table of our intelligent room (see Fig. 1). The reason for that is to keep the privacy of the people as much as possible and to avoid registering images of the user during his daily and private activities.

The visual memory system is composed of two main steps. In the first one changes are detected in the area of interest, the top of the table in our case, which usually correspond to appearance, disappearance or movement of objects. In a second step the areas corresponding to the changes are analyzed and new objects are categorized. The complete visual memory system is shown in Fig. 6 .

\subsection{Change Detection}

The first step of our visual memory is responsible for detecting changes in the area of interest, which is a table in our case. The change detector works as 


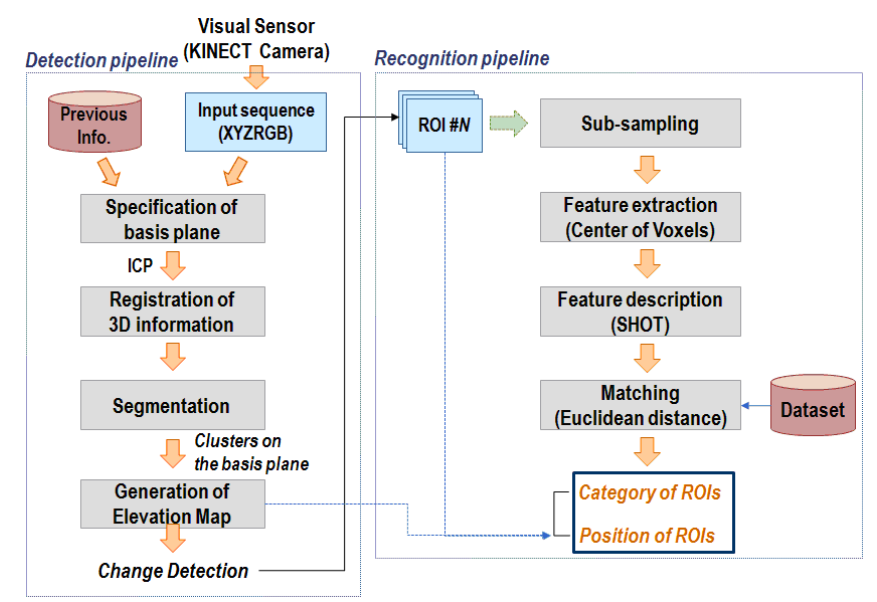

Fig. 6. Schematic diagram for change detection and its categorization.

follows. At some point in time $t_{1}$ the service robot takes a snapshot $z_{1}$ of a table. Since we use a kinect camera then our observation is composed of a 3D point cloud. At some later point in time $t_{2}$ the robot takes a second snapshot $z_{2}$ of the same table. The positions $p_{1}$ and $p_{2}$ of the robot during each observation are known and determined our localization system so that we can situated each observation in a global reference system. In addition, we improve the alignment of the two point clouds using the ICP algorithm. This step allows us to correct small errors that can occur in our localization system.

For each independent view, we extract the plane corresponding to the top of the table by applying a method based on RANSAC. The remaining points, which pertain to the possible objects on top of the table, are projected to a $2 \mathrm{D}$ grid. The cells in this grid are clustered using connected components and each resulting cluster is assumed to be the $2 \mathrm{D}$ representation of a different object on the table. We then compare the 2D clusters in each view and determine the different clusters between the two views which correspond to changes on the table. A resulting change detection is shown in Fig. 7

\subsection{Object Categorization}

The point clusters corresponding to possible changes on the table are further categorized into a set of predefined set of object categories contained in our database as shown in Fig. 8. Our method finds the best matching between the cluster representing a change and the cluster representing each object in our dataset. Our 3D matching method is based on correspondence grouping [?] using the SHOT 3D surface descriptor [?] as key point descriptor. The best matching is obtained as the minimum distance according to 

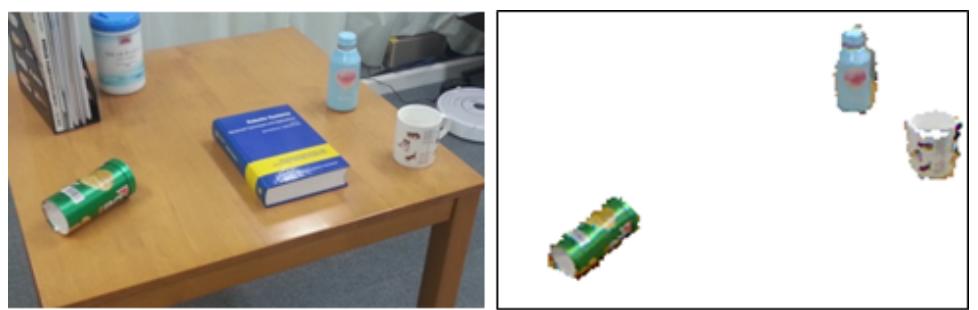

(a) Memory data
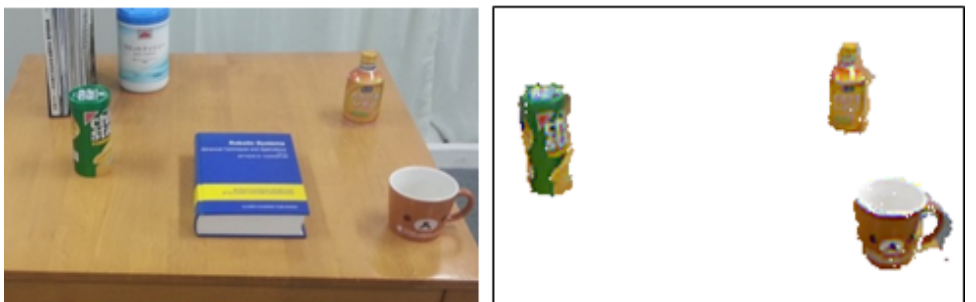

(b) Newly captured data

Fig. 7. Changes detected between two consecutive views of a table.

$$
D=\frac{\operatorname{corr}}{\max \left(N_{\text {model }_{j}}, N_{\text {cluster }}\right)},
$$

where corr represent the number of correspondences between keypoints in the model of our dataset and the keypoints in the cluster, $N_{\text {model }_{j}}$ indicates the number of keypoints found in the model, and $N_{\text {model }}$ represents the number of keypoints found in the cluster.

\subsection{Grasp planning}

Once an object is found in the environment the robot usually needs to grasp it to deliver it to the user. In our system we approximate objects using a polygon model. Using this model the robot should find a feasible grasp posture automatically and quickly. Therefore we use two approximation boxes for both the object and the robot hand. The first box is composed of the bounding box called object convex polygon (OCP) which includes the object in object coordinate system. For large object, we split the object into several regions and calculate the OCP for each region. The second box which means the capacity of object size for the hand is also defined in the hand coordinate system as a grasp rectangular convex (GRC). Using these boxes our planner determines the hand position/orientation by checking whether the position/orientation of GRC can include the OCP. For selecting one grasping posture from multiple candidates of the position/orientation of the hand, we evaluate the movement capability of inverse kinematics solution. 


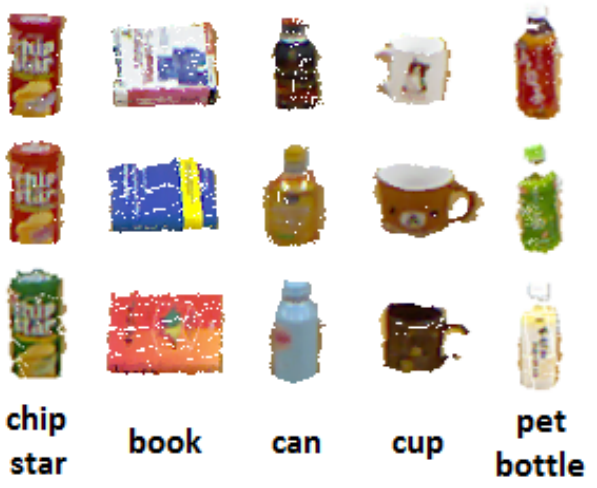

Fig. 8. Dataset of daily life objects for our environment.
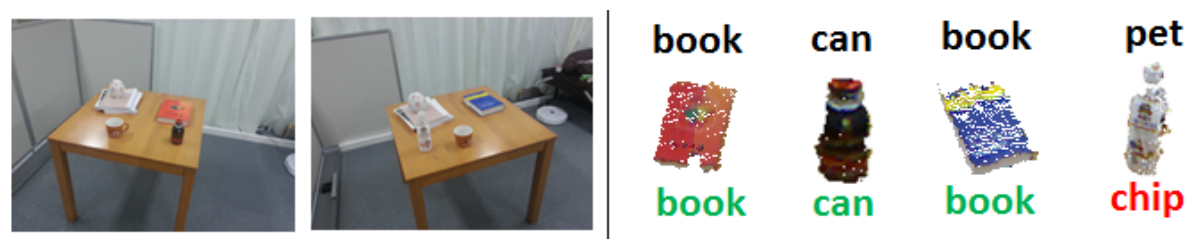

Fig. 9. Resulting matching of changes on a table. The top row indicates the label of the changed objects. The bottom row shows the categorization using our method. The pet bottle is misclassified as a chip container.

\section{Discusion and Future Work}

In this paper we have introduced our informationally structured room which is designed to support daily activities of elder people. The room contains several sensors to monitor the environment and the person. Moreover, the person is assisted by a humanoid robot which uses the information of the environment to support different activities. In addition, we want to stress the importance of keeping the privacy of people during their daily activities and the need to reduce the invasion of their privacy as much as possible.

In this work we have concentrated on the go-and-fetch task which we prognosticate to be one of the most demanding tasks by elderly in their daily life. In this respect we have presented the different subsystems that are implicated in this task, and have showed several independent short-term experiments to demonstrate the suitability of the different subsystems. In the future we aim to design and prepare a long-term experiment in which we can test the complete system for a longer period of time. 

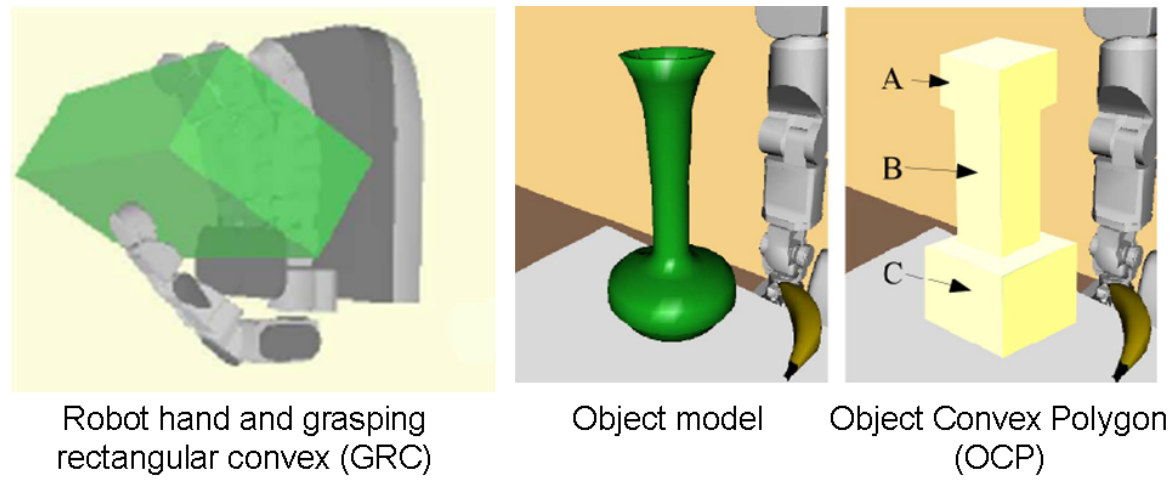

Fig. 10. a grasp rectangular convex for a hand, an object model of a base, and object convex polygons of the model

\section{References}

1. Paolo Dario, Eugenio Guglielmelli, Cecilia Laschi, and Giancarlo Teti. Movaid: A personal robot in everyday life of disabled and elderly people. Technology and Disability, 10:77-93, 1999.

2. T. Hasegawa and K. Muarkami. Robot town project: Supporting robots in an environment with its structured information. In Proc. of the 5th Int. Conf. on Ubiquitous Robots and Ambient Intelligence, pages 119-123, 2006.

3. K. Kawamura and M. Iskarous. Trends in service robots for the disabled and the elderly. In Proc. of the IEEE/RSJ International Conference on Intelligent Robots and Systems (IROS), volume 3, pages 1647-1654, sep 1994.

4. K. Kayama, I.E. Yairi, and S. Igi. Semi-autonomous outdoor mobility support system for elderly and disabled people. In Proc. of IEEE/RSJ Int. Conf. on Intelligent Robots and Systems, pages 2606-2611, 2003.

5. B.K. Kim, N. Tomokuni, K. Ohara, T. Tanikawa, K. Ohba, and S. Hirai. Ubiquitous localization and mapping for robots with ambient intelligence. In Proc. of IEEE/RSJ Int. Conf. on Intelligent Robots and Systems, pages 4809-4814, 2006.

6. Minseong Kim, Suntae Kim, Sooyong Park, Mun-Taek Choi, Munsang Kim, and H. Gomaa. Service robot for the elderly. IEEE Robotics Automation Magazine, 16(1):34-45, march 2009.

7. J.H. Lee and H. Hashimoto. Intelligent space - concept and contents. Advanced Robotics, 16(3):265-280, 2002.

8. T. Mori, A. Takada, H. Noguchi, T. Harada, and T. Sato. Behavior prediction based on daily-life record database in distributed sensing space. In Proc. of IEEE/RSJ Int. Conf. on Intelligent Robots and Systems, pages 1833-1839, 2005.

9. K. Murakami, T. Hasegawa, R. Kurazume, and Y. Kimuro. A structured environment with sensor networks for intelligent robots. In Proc. of IEEE Int. Conf. on Sensors, pages 705-708, 2008.

10. K. Murakami, T. Hasegawa, K. Shigematsu, F. Sueyasu, Y. Nohara, Byong Won Ahn, and R. Kurazume. Position tracking system of everyday objects in an every- 
day environment. In Proc. of the IEEE/RSJ International Conference on Intelligent Robots and Systems (IROS), pages 3712-3718, Oct. 2010.

11. K. Murakami, K. Matsuo, T. Hasegawa, and Kurazume. Position tracking and recognition of everyday objects by using sensors embedded in an environment and mounted on mobile robots. In Proc. of the IEEE International Conference on Robotics and Automation (ICRA), pages 2210 -2216, May 2012.

12. Kouji Murakami, Tsutomu Hasegawa, Ryo Kurazume, and Yoshihiko Kimuro. Supporting robotic activities in informationally structured environment with distributed sensors and rfid tags. Journal of Robotics and Mechatronics, 21(4):453459, 2009.

13. Y. Nakauchi, K. Noguchi, P. Somwong, T. Matsubara, , and A. Namatame. Vivid room: Human intention detection and activity support environment for ubiquitous autonomy. In Proc. of IEEE/RSJ Int. Conf. on Intelligent Robots and Systems, pages 773-778, 2003.

14. Y. Nishida, H. Aizawa, T. Hori, N.H. Hoffman, T. Kanade, and M. Kakikura. 3d ultrasonic tagging system for observing human activity. In Proc. of the IEEE/RSJ International Conference on Intelligent Robots and Systems (IROS), volume 1, pages 785-791, oct. 2003.

15. Y. Nohara, T. Hasegawa, and K. Murakami. Floor sensing system using laser range finder and mirror for localizing daily life commodities. In Proc. of the IEEE/RSJ International Conference on Intelligent Robots and Systems (IROS), pages 1030 -1035 , oct. 2010.

16. C.D. Nugent, D.D. Finlay, P. Fiorini, Y. Tsumaki, and E. Prassler. Home automation as a means of independent living. IEEE Trans. Automation Science and Engineering, 5(1):1-9, 2008.

17. N. Roy, G. Baltus, D. Fox, F. Gemperle, J. Goetz, T. Hirsch, D. Magaritis, M. Montemerlo, J. Pineau, J. Schulte, and S. Thrun. Towards personal service robots for the elderly. Carnegie Mellon University, 2000.

18. S. Srinivasa, D. Ferguson, C. Helfrich, D. Berenson, A. Collet, R. Diankov, G. Gallagher, G. Hollinger, J. Kuffner, and M. VandeWeghe. Herb: A home exploring robotic butler. Autonomous Robots, 28:5-20, 2010. 